\title{
Oxygen clamps in gold nanowires
}

\author{
Frederico D. Novaes ${ }^{1}$, Antônio J. R. da Silva ${ }^{1}$, E. Z. da Silva ${ }^{2}$, and A. Fazzio ${ }^{1}$ \\ ${ }^{1}$ Instituto de Física, Universidade de São Paulo, CP 66318, 05315-970, São Paulo, SP, Brazil \\ ${ }^{2}$ Instituto de Física "Gleb Wataghin", UNICAMP, CP 6165, 13083-970, Campinas, SP, Brazil
}

(Dated: July 11, 2018)

\begin{abstract}
We investigate how the insertion of an oxygen atom in an atomically thin gold nanowire can affect its rupture. We find, using $a b$ initio total energy density functional theory calculations, that $\mathrm{O}$ atoms when inserted in gold nanowires form not only stable but also very strong bonds, in such a way that they can extract atoms from a stable tip, serving in this way as a clamp that could be used to pull a string of gold atoms.
\end{abstract}

Gold nanowires have been at the center of many studies for the past 10 to 15 years 1, 2, 3, 4, 5, 6, 7, 8, 9, 10, $11,12,13,14,15,16,17,18,19,20,21,22,23]$. One of the reasons being that they can form chains that are one atom wide, and a few atoms in length. These chains can be formed in a variety of ways; via a Scanning Tunneling Microscope (STM) tip 3, 4, 6, 7] that contacts a surface and is then retrieved; from a small junction that is mechanically broken 2]; or through the bombardment of electrons that drill holes in a thin gold foil 8,12 . These works are motivated first for the fundamental interest to understand the properties of the ultimate wires, as well as the more applied question of how to use nanowires as electric contacts in nanoeletronic devices. Ultimately, as Feynman pointed out in his seminal speech delivered at the APS 1959 Meeting: "When we get to the very, very small world - say circuits of seven atoms - we have a lot of new things that would happen that represent completely new opportunities for design."

One observed fact is that atomically thin gold nanowires do not usually have more than three to four suspended atoms connecting the leads. In a previous study of the dynamical evolution of a gold nanowire, we investigated through tight-binding molecular dynamics (TB-MD) simulation the formation of such an atomically thin string of atoms all the way up to its rupture 11]. We have found that gold atoms were inserted into the wire from the bulk until the tip connecting the suspended $\mathrm{Au}$ atoms attained a stable configuration. When the force required to extract another atom from the bulk was larger than that necessary to cause the rupture of the wire, it broke.

Moreover, if we start from this final wire configuration (stable tip plus suspended gold atoms), but for a length smaller than the one required to break the wire, and insert impurities such as H, C, C-C, S, N, C-H and C- $\mathrm{H}_{2}$ in between two $\mathrm{Au}$ atoms in the wire, we observe that the $\mathrm{Au}-\mathrm{X}-\mathrm{Au}$ (X being any one of the impurities) bond seems to be stronger than an Au-Au bond, since the wire's rupture always happened at an $\mathrm{Au}-\mathrm{Au}$ bond 18]. The stable tips, however, did not change and as a consequence the wire's number of atoms did not change. As we report below the same is not true for oxygen. It seems that the Au$\mathrm{Au}-\mathrm{O}-\mathrm{Au}-\mathrm{Au}$ bond becomes so strong that upon pulling more gold atoms get extracted from the already stable tip, increasing in this way the wire's length. Therefore, it might be possible to judiciously use gold-oxygen bonds as a clamp to pull longer strings of suspended atoms, longer than in the pure gold case. Ideas along these lines have been proposed for hydrogen [22] and sulfur 24]. These studies are what is being called nanowire chemistry, a new branch of nanocatalysis.
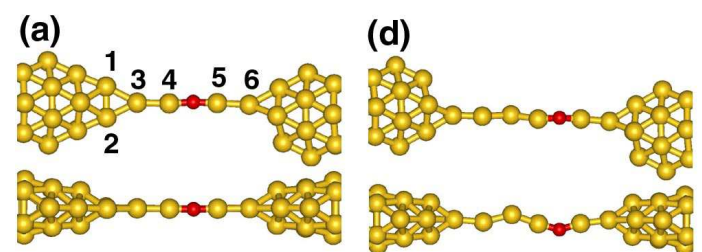

(b)

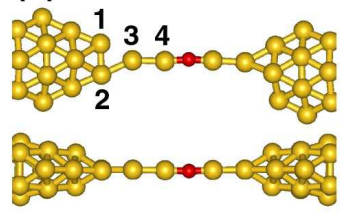

(c)

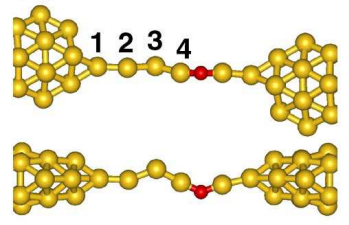

(e)

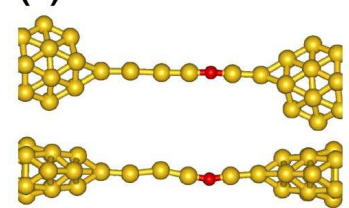

(f)

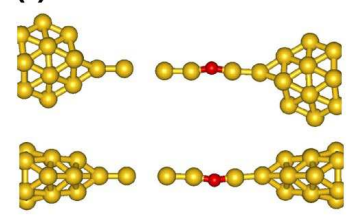

FIG. 1: (Colour online) Evolution of a gold (large spheres) nanowire with an incorporated oxygen (small sphere) atom. Six representative configurations, (a)-(f), are presented. For each configuration two distinct views of the wire are presented. The interatomic distances between labeled atoms are displayed in Table 1.

All our results are based on ab initio total energy density functional theory 25] calculations 26]. We have here employed the same procedure as in the previous previous investigation of the effect of impurities on the breaking of gold nanowires [18, 23]. The oxygen atom was positioned in place of the $\mathrm{Au}$ atom at the center of the chain, forming in this way two Au-O bonds. Therefore, the wire now had $69 \mathrm{Au}$ atoms and one $\mathrm{O}$ atom. After this insertion, all the atoms were again allowed to relax. We then pulled the wire by increasing the supercell length, always allowing the whole system to relax between pulls. This 
protocol was repeated until we observed the full rupture of the wire. It should be stressed that this procedure uses a realistic structure for the nanowire, in particular where the impurity atom is placed, considers the wire under stress, and allows the system to break either at an $\mathrm{Au}-\mathrm{Au}$ bond, as in a clean wire, or at an $\mathrm{Au}-\mathrm{O}-\mathrm{Au}$ bond.

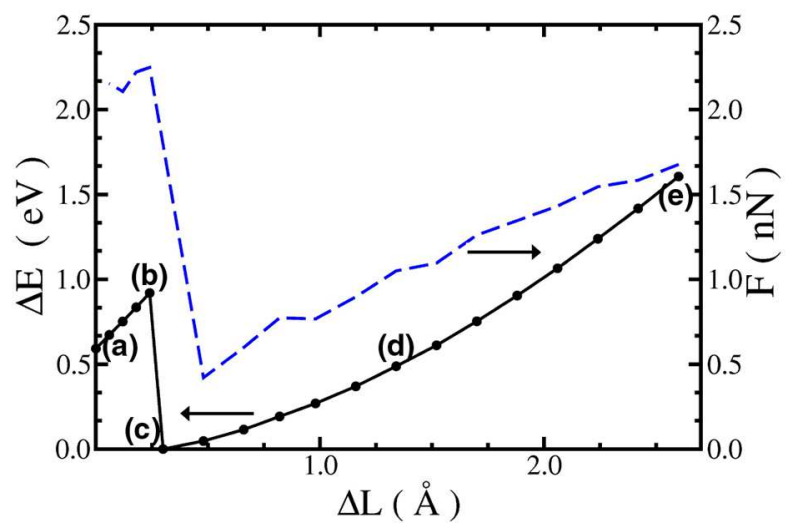

FIG. 2: Total energy (lower curve; left axis) and calculated pulling force acting on the nanowire (upper curve; right axis) along the stages of the simulation. The total energy values are displayed relative to the lowest energy structure, shown in Fig. 1(c). The results are presented as a function of the displacement $\Delta \mathrm{L}$ of the wire. The configurations presented in Fig. 1 are marked accordingly in the graph.

The evolution of the gold nanowire with the incorporated oxygen atom may be seen in Figure 1, where we present six representative structures. As can be seen from Fig. 1(a), the atomically thin neck had originally four suspended gold atoms (labeled from 3 to 6 ). The oxygen atom was inserted in the middle of the neck, between atoms 4 and 5. The interatomic distances are presented in Table I. From the distances between the Au atoms 1 and $3(3.06 \AA)$ and between the atoms 2 and $3(2.97 \AA)$ in the configuration shown in Fig. 1(a), and based on simulations 18, 21] where we noticed that the wire tends to break when there is an $\mathrm{Au}-\mathrm{Au}$ distance close to 3.0-3.1 $\AA$, we could expect that the wire would break. Instead of breaking, however, upon further pulling of the wire we have observed the rearengement of the tip, with the removal of two atoms (atoms labeled 2 and 3). It can be seen from Fig. 1(b) the breaking of the bond between atoms 1 and 3, and subsequently (Fig. 1(c)) the gold atom 2 being also pulled from the tip into the neck. This occured concomitantly with the restructuring of the tip, with the displacement of atom 1 , as can be observed in Fig. 1(c). One should note that oxygen caused all this sequence of events even though the original geometry of the tip has been shown 11, 21] to be quite stable, a fact that happened only for the $\mathrm{O}$ atom among all the impurities that we have studied [18, 20] so far. This means that the oxygen impurity modifies and strengthens the neck significantly, which is most likely related to strong $\mathrm{Au}-\mathrm{O}$ bonds that are formed in low dimensional Au structures 16.

As a result of the insertion of more $\mathrm{Au}$ atoms in the

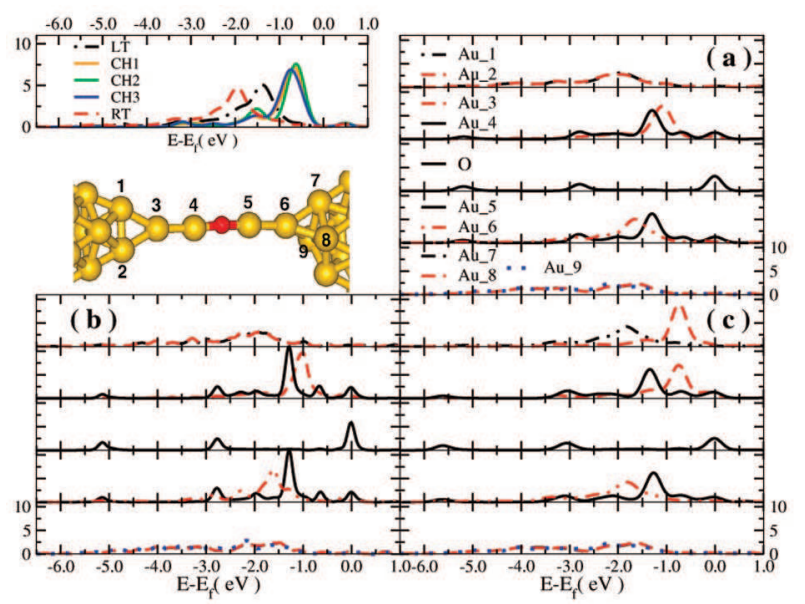

FIG. 3: (Colour online) Projected DOS (PDOS - arbitrary units) for the nine labeled gold atoms shown in the left middle panel, plus the oxygen. In the top left panel we present the PDOS for the pure gold nanowire shown in Fig. 4(d). LT and RT stand for left and right tip connecting atoms, respectively, whereas $\mathrm{CH} 1, \mathrm{CH} 2$ and $\mathrm{CH} 3$ correspond to the first, second and third chain atoms, from left to wright, respectively. The results shown in figures (a) through (c) correspond to the structures presented in Fig. 1(a), Fig. 1(b), and Fig. 1(e), respectively.

TABLE I: Interatomic distances (in $\AA$ ) between Au atoms labeled from 1 to 6 in Fig. 1, for selected snapshots (from Fig. 1(a) through Fig. 1(f)). The boldface distance for structure (f) indicates the bond that breaks.

\begin{tabular}{ccccccc} 
Structure & $1-2$ & $1-3$ & $2-3$ & $3-4$ & $4-5$ & $5-6$ \\
\hline (a) & 2.71 & 3.06 & 2.97 & 2.82 & 4.20 & 2.82 \\
(b) & 2.71 & 3.17 & 2.99 & 2.84 & 4.21 & 2.84 \\
(c) & 2.65 & - & 2.66 & 2.59 & 3.70 & 2.61 \\
(d) & 2.71 & - & 2.73 & 2.63 & 3.89 & 2.66 \\
(e) & 2.79 & - & 2.98 & 2.69 & 4.07 & 2.73 \\
(f) & 2.63 & - & $\mathbf{3 . 8 6}$ & 2.58 & 3.95 & 2.66
\end{tabular}

neck, there is an overall stress release in the system. This can be clearly seen in Fig. 2 where we present both the total energy as well as the calculated pulling force acting on the nanowire. This is the expected behavior at this stage of nanowire stretching [10], namely, an elastic stage with linear increase of the force, followed by a sudden release of the tension related to the tip reconstruction. Fig. 1 and 2 show exactly that. From a structural point of view, what happened after the tip reconstruction was the formation of a zig-zag structure in the neck, as can be seen in Fig. 1(c). This is a consequence of the smaller stress that is being applied to the neck region as a result of the insertion of more atoms in the neck. During the elastic stage following the stress release, there is a "linearization" of the neck, that continues all the way up to the rupture point, that occurs between atoms 2 and 3 . This happens for a pulling force of approximately $1.7 \mathrm{nN}$, 


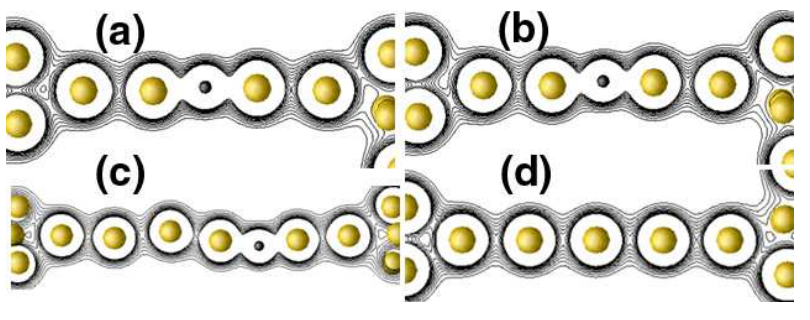

FIG. 4: (Colour online) Contour plots for the total electronic charge density, in planes that pass through the neck atoms. The results shown in figures (a) through (c) correspond to the structures presented in Fig. 1(a), Fig. 1(b), and Fig. 1(e), respectively. In (d) we present a similar plot for a pure gold nanowire prior to its rupture.

which is in good agreement with the experimental value of $1.5 \pm 0.3 \mathrm{nN}$ measured for a pure gold nanowire 10 . This value for the rupture force is what one would expect for the breaking of an $\mathrm{Au}-\mathrm{Au}$ bond. This can be compared to the value of $2.3 \mathrm{nN}$ of the force that causes the tip reconstruction, indicating that the whole neck, including oxygen second nearest neighbor pure $\mathrm{Au}-\mathrm{Au}$ bonds, are strengthened by the presence of the $\mathrm{O}$ atom.

In order to gain insight into the electronic structure of the $\mathrm{Au}$ nanowires with the $\mathrm{O}$ impurity, and how it evolves as the wire is pulled, we present in Fig. 3 the projected density of states (PDOS). The PDOS is shown for all gold atoms labeled from 1 to 9 in the left middle panel of Fig. 3, which encompasses all the neck atoms as well as some of the tip atoms. The PDOS for the oxygen atom is also shown. In Fig. 3 (top left panel) we also present, for comparison, the PDOS for a pure gold nanowire (see Fig. 4 for the atomic structure). In Fig. 3 (a) we show the PDOS for the configuration shown in Fig. 1(a). For this configuration the tip atoms (atoms $1,2,7,8$, and 9) have an electronic structure which is similar to bulk gold, as we had already observed before in pure nanowires 21. Again, similarly to the pure gold nanowire, for the neck atoms (atoms 3-6) that have a smaller number of neighbors, we observe: (i) that their PDOS become narrower; (ii) are characterized by sharper peaks; and (iii) present an overall shift towards higher energies. The fact that the Au PDOS has an overall shift towards lower energies as the number of nearest neighbors increases can be clearly seen when one compares the results for atoms 3 and 6 , that connect the tip to the neck. Atom 3 has three neighbors whereas atom 6 has four neighbors. This is reflected in a small shift of the atom 6 PDOS towards lower energies when compared to the atom 3 PDOS. This can be also seen in the pure gold nanowire PDOS. Finally, the oxygen atom PDOS has a clear peak at the Fermi energy. The $\mathrm{O}$ two gold neighbor atoms also have peaks at the Fermi energy, a result of the chemical bonding and orbital hybridization. In fact, the PDOS of these two Au atoms (atoms 4 and 5 ) are very similar. An important feature related to the wire's stability is the peak at approximately $5 \mathrm{eV}$ below the Fermi level. From charge density plots (not shown) it is possible to see that this peak is associated with a $O_{p}-A u_{d}$ bonding orbital. This orbital clearly involves the oxygen atom and its two Au nearest neighbors, however, it also has some weight from the oxygen Au second nearest neighbors. These peaks are clearly absent in the pure Au PDOS, indicating that besides the delocalized, metal-like bonding that stabilizes these $\mathrm{Au}$ wires, the local, covalent-like bonding between the $\mathrm{Au}$ and $\mathrm{O}$ have a significant contribution in the strength increase that we observe.

In Fig. 3(b) we present the PDOS for the configuration shown in Fig. 1(b). As the wire is stretched, there is a sharpening of the projected DOS of the neck atoms, in particular for atom 3, since it is evolving from a threeatom coordination to a two-atom coordination. There are no significant alterations in the PDOS for the tip atoms. In particular, the PDOS for the tip atoms 7, 8, and 9 do not suffer any significant changes throughout the evolution of the wire, since their environment does not get altered. The PDOS for the configuration shown in Fig. 1(e) is presented in Fig. 3(c). Most of the features already discussed are also present. The only noticeable changes when compared to Fig. 3(a) are the significant modifications in the PDOS of atom 2. As this atom is now a neck atom, its PDOS is shifted towards higher energies, and develops a sharper peak when compared to a more bulk like atom.

In Fig. 4 we show contour plots for the total electronic charge density, in planes that pass through the neck atoms. Fig. 4(a) corresponds to the structure presented in Fig. 1(a). The charge density for the Au-O bonds when compared to the value for the pure $\mathrm{Au}-\mathrm{Au}$ bonds indicate that oxygen is in fact making strong bonds with the gold atoms. This feature is preserved as the wire evolves, as can be seen in Fig. 4(b), which corresponds to the structure presented in Fig. 1(b), and in Fig. 4(c), which corresponds to the structure presented in Fig. 1(e). Moreover, there is also an increase of the charge density between the $\mathrm{Au}-\mathrm{Au}$ bonds closest to the oxygen atom (between atoms 3 and 4 and between atoms 5 and 6 ). Therefore, it seems that the oxygen atoms is affecting the wire in such a way that the structure $\mathrm{Au}$ $\mathrm{Au}-\mathrm{O}-\mathrm{Au}-\mathrm{Au}$ becomes strong. For comparison, we show in Fig, 4(d) similar contour plots for a pure gold nanowire prior to its rupture. From Fig. 4(a) and Fig. 4(b) we can see that the bond between atoms 1 and 3 is becoming weaker, which is consistent with the restructuring of the tip. Also, from Fig. 4(c) the weaker bond is between atoms 2 and 3, which is precisely where the wire breaks.

From all these results we can rationalize some general features: (i) even though there is a metallic, delocalized character in the bonds at the nanowire, they also have a rather localized nature involving nearest neighbor atoms. This is evident both from the fact that gold atoms with similar number of neighbors have similar PDOS and from the observation that the impurity affects primarily the PDOS of its nearest neighbors. Also, there are clearly 
$\mathrm{Au}-\mathrm{O}-\mathrm{Au}$ bonding orbitals that show up as low energy peaks in the PDOS; (ii) the rupture of the wire usually happens at bonds involving atoms whose PDOS are displaced towards higher energy values, closer to the Fermi energy, which is related to a weakening of these bonds. See, for example, the PDOS for atoms 2 and 3 in Fig. 3 (c). In particular, the PDOS for atom 3 is also closer to the Fermi energy in Fig. 3(a), and the first bond that breaks at the tip reconstruction is precisely between atoms 3 and 1 . However, the PDOS for atom 1 is still delocalized because it has a higher coordination number; (iii) the oxygen atom has an influence that extends towards its next-nearest neighbors. This is why the bond between atoms 3 and 4 does not break. Instead, the neck bonds become so strong that the pulling force attains a value of $2.3 \mathrm{nN}$, which is suficcient to disrupt the tip.

In conclusion, we have presented state-of-the-art calculations of a realistic Au atomic chain nanowire with an inserted oxygen impurity atom. In particular, we have investigated how the $\mathrm{O}$ atom can affect the rupture of the wire. We find that $\mathrm{O}$ atoms can form not only stable but also very strong bonds with the Au atoms, in such a way that they can extract atoms from a stable tip. This indicates that in an oxygen reach atmosphere it may be possible to pull a longer string of gold atoms 31. If one considers that in most situations wires with more than, say, 4-5 atoms have not been observed, the probable reason being that stable tips are quickly formed and $\mathrm{Au}-\mathrm{Au}$ bonds do not sustain forces greater than approximately $1.8 \mathrm{nN}$, this opens up the possiblity to study: (i) if this local bond strength interpratation is valid; (ii) how stable can tips be; and (iii) the general stability properties of Au nanowires. Finally, as the $\mathrm{O}$ atom has a clear peak at the Fermi energy, it will be interesting to see how it affects the charge transport properties of the nanowire. Such an investigation is currently under way.

We thank J. M. van Ruitenbeek for communicating unpublished work. We acknowledge support from FAPESP, Capes and CNPq.
[1] U. Landman, W. D. Luedtke, N. A. Burnham, and R. J. Colton, Science 248, 454 (1990).

[2] C. J. Muller, J. M. van Ruitenbeek, and L. J. de Jongh, Phys. Rev. Lett. 69, 140 (1992).

[3] N. Agraï, J. G. Rodrigo, and S. Vieira, Phys. Rev. B 47, R12345 (1993).

[4] G. Rubio, N. Agraït, and S. Vieira, Phys. Rev. Lett 76, 2302 (1996).

[5] U. Landman, W. D. Luedtke, B. E. Salisbury, and R. L. Whetten, Phys. Rev. Lett. 77, 1362 (1996).

[6] H. Ohnishi, Y. Kondo, and K. Takayanagi, Nature 395, 780 (1998).

[7] A. I. Yanson, et al., Nature 395, 783 (1998).

[8] Y. Kondo and K. Takayanagi, Phys. Rev. Lett. 79, 3455 (1999)

[9] H. Häkkinen, R. N. Barnett, A. G. Scherbakov, and U. Landman, J. Phys. Chem. B 104, 9063 (2000).

[10] G. Rubio-Bollinger, S. R. Bahn, N. Agrait, K. W. Jacobsen, and S. Vieira, Phys. Rev. Lett. 87, 026101 (2001).

[11] E. Z. da Silva, A. J. R. da Silva, and A. Fazzio, Phys. Rev. Lett. 87, 256102 (2001).

[12] V. Rodrigues and D. Ugarte, Phys. Rev. B 63, 073405 (2001).

[13] Y. Takai, T. Kawasaki, Y. Kimura, T. Ikuta, and R. Shimizu, Phys. Rev. Lett. 87, 106105 (2001).

[14] S. B. Legoas , D. S. Galvão , V. Rodrigues, and D. Ugarte, Phys. Rev. Lett. 88, 076105 (2002).

[15] C.Untiedt, A. I. Yanson, R. Grande, G. Rubio-Bollinger, N. Agraï, S. Vieira, and J. M. van Ruitenbeek, Phys. Rev. B 66, 085418 (2002).

[16] S. R. Bahn, N. Lopez, J. K. Norskov, and K. W. Jacobsen, Phys. Rev. B 66, 081405(R) (2002).

[17] N. Agraït, A. L. Yeyati, and J. M. van Ruitenbeek, Phys. rep. 377, 81 (2003).

[18] F. D. Novaes, A. J. R. da Silva, E. Z. da Silva, and A. Fazzio, Phys. Rev. Lett. 90036101 (2003).

[19] N. V. Skorodumova and S. I. Simak, Phys. Rev. B 67, 121404(R) (2003).
[20] E. Z. da Silva, A. J. R. da Silva and A. Fazzio, Comput. Mat. Sci. 30, 73 (2004).

[21] E. Z. da Silva, F. D. Novaes, A.J.R. da Silva, and A. Fazzio, Phys. Rev. B 69, 115411 (2004).

[22] R. N. Barnett, H. Häkkinen, A. G. Scherbakov, and U. Landman, Nano Lett. 4, 1845 (2004).

[23] F. D. Novaes, E. Z. da Silva, A. J. R. da Silva, and A. Fazzio, Surf. Sci. 566-568, 367 (2004).

[24] D. Kruger, R. Rousseau, H. Fuchs, and D. Marx, Angew. Chem.-Int. Ed. 42, 2251 (2003).

[25] P. Hohenberg and W. Kohn, Phys. Rev 136, 864B (1964); W. Kohn and L. J. Sham, Phys. Rev. 140, 1133A (1965).

[26] We have used a GGA approximation 27]; normconserving pseudopotentials 28]; periodic-boundary conditions; a supercell approximation with lateral separation of $20 \AA$ between wires; and the X-point for the Brillouin zone sampling. All calculations were performed using the SIESTA code 29], and we employ a DZP basis function with a confining energy shift of $0.07 \mathrm{eV}[30]$ and a cutoff of $250 \mathrm{Ry}$ for the grid integration. The procedure of elongation and atomic relaxation was the same as in [18]. Even though we report non-spin polarized calculations, we have performed tests including spin polarization for geometries close to the tip reconstruction point, and none of the main conclusions have changed.

[27] J. P. Perdew, K. Burke, and M. Ernzerhof, Phys. Rev. Lett. 77, 3865 (1996).

[28] N. Troullier and J. L. Martins, Phys. Rev. B 43, 1993 (1991).

[29] P. Ordejón, E. Artacho, and J. M. Soler, Phys. Rev. B 53, R10441 (1996); D. Sánchez-Portal, P. Ordejón, E. Artacho, and J. M. Soler, Int. J. Quantum Chem. 65, 453 (1997).

[30] E. Artacho, D. Sánchez-Portal, P. Ordejón, A. Garcia, and J. M. Soler, Phys. Status Solidi B 215, 809 (1999).

[31] It came to our knowledge, after submission of our work, that experiments show in fact this behavior. See W. H. A. Thijssen, D. Marjenburgh, R. H. Bremmer, and J. M. 
van Ruitenbeek, cond-mat/0509376 